\title{
The Role of Students' Gender and Anxiety in Physics Performance
}

\author{
Elise Agra ${ }^{1}$, Susan M. Fischer ${ }^{2}$, and Sian L. Beilock ${ }^{1}$ \\ ${ }^{1}$ Department of Psychology, University of Chicago, 5848 S. University Ave., Chicago, IL, 60637 \\ ${ }^{2}$ Department of Physics, DePaul University, 2219 N. Kenmore Ave., Chicago, IL, 60614
}

\begin{abstract}
We investigated the relation between students' physics anxiety and their physics performance, and whether this relation differs as a function of gender. To measure students' levels of physics anxiety, we used the Physics Anxiety Rating Scale, which contains 32 items in four categories: course/test anxiety, anxiety about the lack of physics knowledge, math anxiety, and physics laboratory anxiety. Reliability analysis using 76 algebra-based introductory physics students showed a high level of internal consistency for the entire scale as well as for each of the four categories. Females reported significantly higher levels of physics laboratory anxiety than males. No other significant gender differences in anxiety levels were observed across the other three categories. In terms of the relation between physics anxiety and performance, course/test anxiety was significantly negatively correlated with the midterm exam for females but not for males, while lack of physics knowledge anxiety was significantly negatively correlated with both the midterm and final exams for the males but not for the females. Finally, we found a significant positive relation between physics laboratory anxiety and the final exam for the males but not for the females, even though females reported significantly higher levels of physics lab anxiety than males.
\end{abstract}

\section{INTRODUCTION}

It is widely established in physics education research literature that male students significantly outperform female students on concept inventories (e.g., ref. [1] and references within). Factors that may explain the gender differences in physics performance include differences in students' previous math and physics background, and their attitudes and beliefs about science [2]. Different instructional strategies such as interactive engagement [3], shifting to studio-style classrooms [4], and even the use of a psychological intervention [5] have been shown to lessen this gender gap.

Here we explore another factor that might be relevant to students' physics performance: physics anxiety. While there have been a large number of studies investigating anxiety including math anxiety, few have dealt with science anxiety, and even fewer with physics anxiety.

Science and math anxiety are characterized by fear or apprehension about science or math concepts, or science- or math-related activities [6, 7]. Functional magnetic resonance imaging (fMRI) studies have shed light on the neural correlates of math anxiety and have shown that when simply anticipating a math task, the higher one's math anxiety, the more one shows increased activity in neural regions associated with visceral threat detection, and often the experience of pain itself $[8,9]$. Math anxiety has also been shown to typically be higher in females than in males and is correlated with poor math performance [10]. Similarly, females tend to experience higher levels of science anxiety than males [11], and the best predictors of science anxiety are non-science anxiety, gender, and choice of major [1112]. Moreover, a semester of physics was shown to reduce students' acute science and non-science anxiety [13].

The development and validation of the Physics Anxiety Rating Scale [14] is recent, and has shown that physics anxiety is higher in females than in males, and is related to students' GPA, major, and type of high school attended [15]. Students' affective characteristics related to physics, which included, among other factors, physics anxiety, also significantly influenced their physics achievement [16].

We investigated the relation between physics anxiety and physics performance (as measured by students' midterm and final exam scores), and whether the relation differs as a function of gender.

\section{METHODS}

\section{A. Physics Anxiety Rating Scale}

The Physics Anxiety Rating Scale (PARS) [14-15] is composed of a set of 32 items scored on a 5-point Likert-type scale ranging from 1 (strongly disagree) to 5 (strongly agree). The items are grouped into four categories. Physics course/test anxiety (CTA) is related to the anxiety about being in a physics class or studying for a physics exam, and includes statements such as "I am usually very nervous when I am studying for a physics exam." Anxiety about the lack of physics knowledge (LPKA) is related to students' perception of their own understanding of physics concepts. An example of an LPKA statement is "When the instructor is solving a problem, I worry that other students understand the solution better than I do." Math anxiety (MA, "Trying to read a sentence full of physics equations that have symbols I don't understand scares me a lot.") is related to students' anxiety about succeeding in the course due to lack of math skills. Finally, physics laboratory anxiety (PLA, "I am very comfortable with lab materials.") is related to students' anxiety about performing an equipment-related activity (i.e., a lab experiment in traditional classrooms).

\section{B. Population and Sample}

Participants in the study were enrolled in one of four sections of the first course of a year-long sequence of 
algebra-based introductory physics $(\mathrm{N}=76 ; 25$ male $)$ at DePaul University. The total number of students in the course was 172 (60\% female).

The course was taught in studio style with interactive engagement and no separate lab meetings. Hands-on and laboratory-type activities were introduced within the regular class meetings, where students worked in groups of three.

\section{Data Collection and Analysis}

The original instrument was developed and administered in Turkish and then was translated to English after the validation studies for publication. Moreover, the survey was created for implementation in a traditional physics classroom (with separate lecture and laboratory classes). To better match our students' experiences in the course (as we don't have traditional lab sections), we made minor modifications to some of the physics anxiety statements, mostly those related to the lab.

Students who provided consent were asked to complete the PARS online. In addition to the physics anxiety measure, students' gender data, midterm and final exam scores were also collected. After collecting the PARS data, the statements were recoded such that a participant's rating reflected her or his degree of anxiety. That is, all items were scored such that the higher the response value the greater the physics anxiety.

\section{RESULTS}

\section{A. Scale Reliability}

First, we tested if the items in our survey measured the same construct (feeling of anxiety) by computing Cronbach's alpha for the four categories and for the whole instrument (Table I). Cronbach's alpha usually ranges between 0 and 1 , and provides a measure of internal consistency of a scale - the closer the coefficient is to 1 , the greater the internal consistency of the items. For the four categories of the PARS, the reliability values ranged from 0.87 to 0.94 , while the whole PARS scale had a value of 0.97 . These coefficient values are considered good $(\alpha \geq 0.8)$ to excellent $(\alpha \geq 0.9)$, and indicate a high level of internal consistency for our scale.

\section{B. Performance Differences}

While students in the four sections used the same in-class worksheets, each instructor gave different midterm and final exams. Thus, we need to check that (1) the midterm and final exam scores did not differ by instructor, and (2) the average midterm and final exam scores of the students who consented to do the survey did not differ from that of the entire class.

To check the first, we used a one-way ANOVA (analysis of variance) to compare the average score of the three instructors' students in the midterm and final exams. We found no significant instructor dependence of both the midterm exam, $F(2,73)=0.21, p=.809$ and the final exam $F(2,73)=0.79, p=.460$.
TABLE I. Number of items and reliabilities for the categories in the PARS.

\begin{tabular}{lcc}
\hline \hline & $\begin{array}{c}\text { \# of } \\
\text { items }\end{array}$ & $\begin{array}{c}\text { Cronbach's } \\
\text { alpha }\end{array}$ \\
\cline { 2 - 3 } CTA (Course/Test) & 9 & 0.94 \\
LPKA (Lack of Physics & 8 & 0.87 \\
Knowledge) & & \\
MA (Math) & 8 & 0.89 \\
PLA (Physics Laboratory) & 7 & 0.87 \\
Whole PARS & 32 & 0.97 \\
\hline \hline
\end{tabular}

Next, we compared the average exam scores of the students in our sample to that of the whole class. Results of the one-sample t-test showed no significant differences $(p>$ .05 ) between the consented students and the whole class on the midterm and final exams, both when looking at all participants and when looking by instructor.

\section{Relationship between Gender, Physics Anxiety, and Performance}

\section{Gender differences in physics anxiety}

An independent samples t-test was conducted to examine the relationship between overall physics anxiety and gender (Fig. 1), in which we found no significant difference between male and female students, $t(74)=0.98$, $p=.329$. We also investigated if the levels of anxiety across the four categories depended on gender. The category $x$ gender interaction was marginally significant, $F(3,222)=$ $2.50, p=.060$. To probe this marginal interaction, we compared the anxiety levels of male students to female students on the four categories of PARS (Fig. 1). There were no gender differences on the course/test anxiety $(t(74)$ $=0.59, p=.555)$, anxiety about the lack of physics knowledge $(t(74)=1.19, p=.239)$, and mathematics anxiety $(t(74)=0.08, p=.934)$. Females reported significantly higher levels of physics laboratory anxiety than males $(t(74)=2.04, p=.045)$.

The gender difference observed in physics laboratory anxiety may be related to results obtained using the Colorado Learning Attitudes about Science Survey for Experimental Physics (E-CLASS), in which males reported significantly higher average scores (i.e., more expert-like attitudes) than females [17]. For instance, it is possible that the PARS physics laboratory anxiety is correlated with responses to all or a subset of questions in E-CLASS.

\section{Effects of physics anxiety and gender on performance}

Here we used multiple linear regression to test if students' physics anxiety and gender predicted their midterm or final exam scores. First, we investigated whether gender, overall physics anxiety, and the interaction between gender and overall physics anxiety predicted students' midterm or final exam scores. 


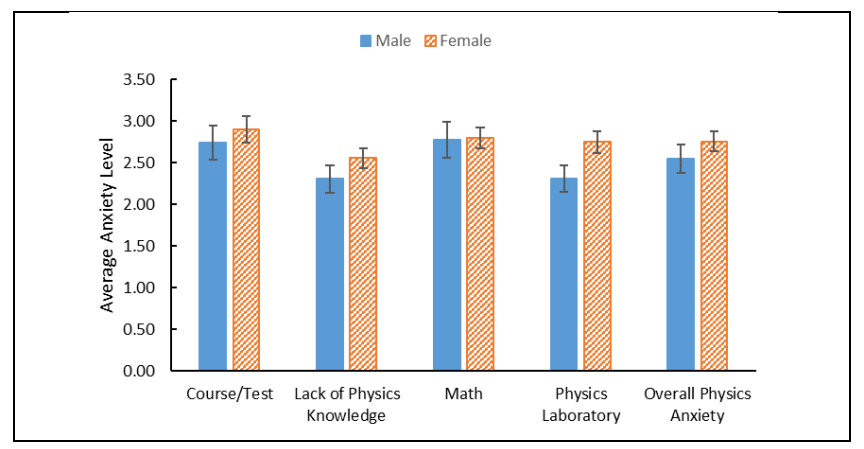

FIG. 1. Anxiety levels of male and female students.

We found a significant effect of physics anxiety on both the midterm exam score $(\beta=-.629, p<.001)$ and the final exam score $(\beta=-.310, p=.017)$. The effect of the interaction between gender and overall physics anxiety on either the midterm or the final exam was not significant.

Next, we used gender, the four anxiety categories (course/test anxiety, lack of physics knowledge anxiety, math anxiety, and physics laboratory anxiety), and the interaction between gender and CTA, LPKA, MA, and PLA to predict students' midterm or final exam scores.

We found a significant interaction between gender and the course/test anxiety ( $\beta=.412, p=.049)$ on the midterm exam such that course/test anxiety had no significant effect on the midterm exam for male students ( $\beta=.044, p=.897$ ), while female students with higher levels of course/test anxiety had lower midterm exam scores $(\beta=-.723, p<.001)$. Figure 2 (top panel) shows the midterm exam scores for female and male students at low (1 SD below the mean), mean, and high (1 SD above the mean) values of course test anxiety. At low levels of course/test anxiety, male and female students' midterm scores are not significantly different. However, high levels of course/test anxiety may have caused female students to underperform while male students don't let their anxiety affect their exam performance. Further research is recommended.

There was also a significant effect of the interaction between gender and lack of physics knowledge anxiety on the midterm exam score $(\beta=-.507, p=.037)$. Figure 2 (bottom panel) shows male and female students' midterm exam scores at low (1 SD below the mean), mean, and high (1 SD above the mean) values of lack of physics knowledge anxiety. Lack of physics knowledge anxiety did not have an effect on female students' midterm exam score $(\beta=-.057, p$ $=.788$ ), while males with higher levels of lack of physics knowledge anxiety had lower scores on the midterm exam ( $\beta$ $=-.980, p=.012$ ).

Analysis on the final exam showed a significant effect of the interaction between gender and lack of physics knowledge anxiety $(\beta=-.785, p=.005)$. For female students, lack of physics knowledge anxiety did not have a significant effect on their final exam scores $(\beta=.053, p=.825)$. On the other hand, male students with higher levels of lack of physics knowledge anxiety had lower final exam scores $(\beta=$
$-1.376, p=.002$ ). Figure 3 (top panel) shows the final exam scores of males and females at low (1 SD below the mean), mean, and high (1SD above the mean) values of lack of physics knowledge anxiety.

The lack of physics knowledge anxiety - performance relationship being present in men but not in women for both the midterm and final exams (bottom panel of Fig. 2 and top panel of Fig. 3) may be an indication that male students are more accurate at assessing their actual lack of physics knowledge. That is, when men are highly anxious about how much they don't know, they perform poorly because they lack knowledge of physics content. On the other hand, the lack of significant correlation for female students could mean that females tend to have anxiety about their lack of physics knowledge regardless of how much physics content they actually know (i.e., they don't trust themselves to accurately evaluate their true level of knowledge).

The interaction between gender and physics laboratory anxiety also had a significant effect on the final exam score ( $\beta=.713, p=.001)$. Female students' physics laboratory anxiety did not significantly affect their score on the final exam ( $\beta=.070, p=.676$ ), while male students with higher levels of physics laboratory anxiety also had higher scores on the final exam $(\beta=1.433, p<.001)$.

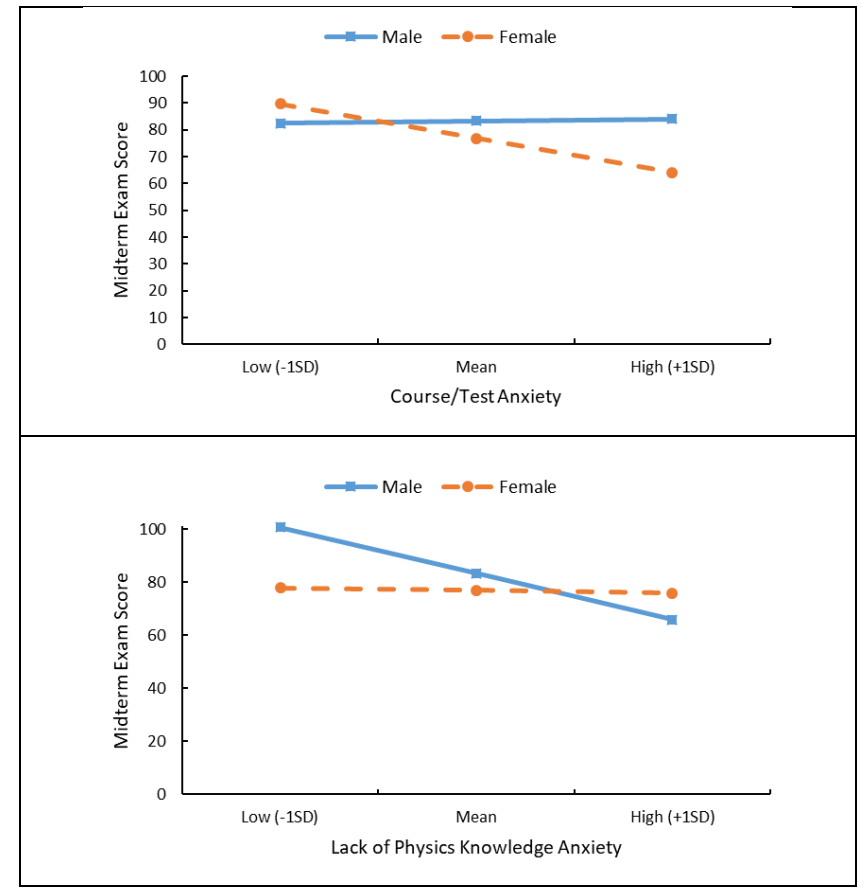

FIG. 2. Simple slopes for the gender x CTA (top) and gender x LPKA (bottom) interactions. Midterm exam scores were plotted at low (1 SD below the mean), mean, and high (1 SD above the mean) anxiety values for male and female students. 


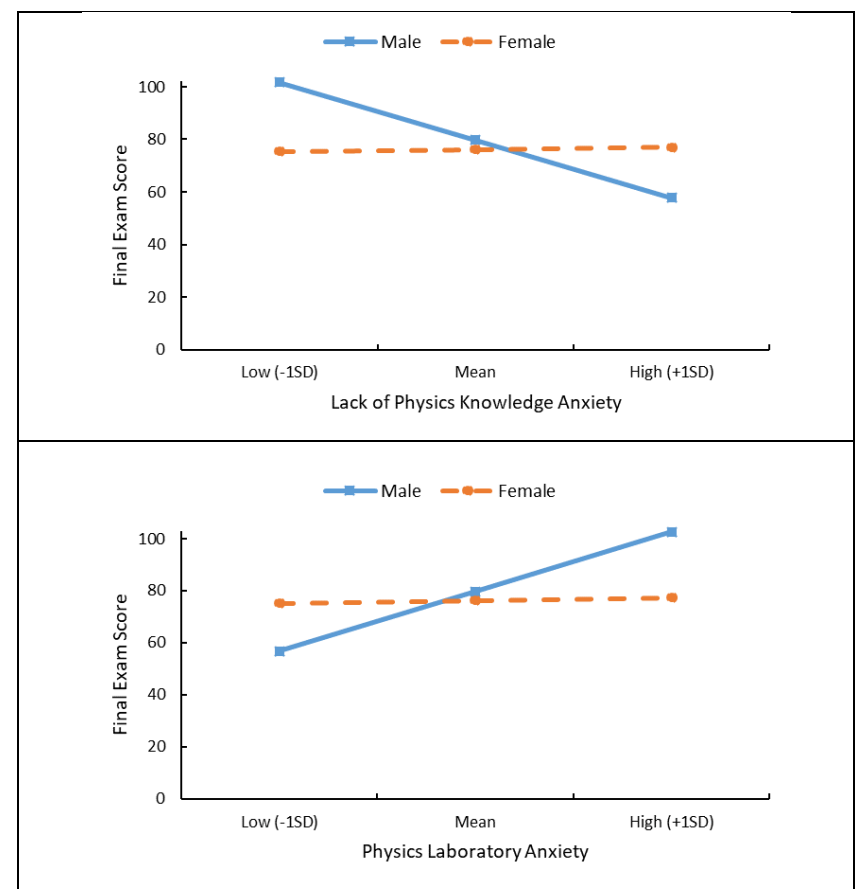

FIG. 3. Simple slopes for the gender x LPKA (top) and gender x PLA (bottom) interactions. Final exam scores were plotted at low (1 SD below the mean), mean, and high (1 SD above the mean) anxiety values for male and female students.

Figure 3 (bottom) panel shows the final exam scores of female and male students at low (1 SD below the mean), mean, and high (1 SD above the mean) values of physics laboratory anxiety. At low physics laboratory anxiety levels, females outperform males on the final exam. However, males score higher than females at high levels of physics laboratory anxiety.

It is unclear how this result should be interpreted, and how best to consider the relationship between students' attitudes and anxiety about lab activities and their performance on tests that stress conceptual understanding and problem solving rather than lab skills and data analysis, as is typical in midterm and final exams for introductory physics courses. In our studio physics classrooms, courses are designed to leverage the use of active engagement and numerous hands-on, laboratory-like activities to enhance students' understanding of physics concepts. It may be that the active engagement aspect of this teaching approach serves to reduce the gender gap, while the tendency for female students to display higher physics lab anxiety may imply a potential negative aspect of this approach, or at least an aspect that requires further study.

\section{CONCLUSIONS}

We evaluated the reliability of the Physics Anxiety Rating Scale, and showed a high level of internal consistency for the entire scale and its four components. We then investigated the effects of students' anxiety and gender on their performance on the midterm and final exams. Overall physics anxiety significantly predicted the midterm and final exam scores such that those who were more highly anxious had lower scores. Course/test anxiety was significantly related to the midterm exam performance in women but not for men, while anxiety about the lack of physics knowledge was significantly correlated with both the midterm and final exam scores for men but not for women. For these interactions, those who reported higher levels of anxiety had lower exam scores. Interestingly, physics laboratory anxiety was significantly positively related to performance in men but not for women, even though women reported significantly higher levels of PLA than men.

Our study has several limitations, and did not consider other gender identities or collect data on factors such as race, that may account for differences in performance. Suggestions for future research also include replicating the current study and correlating physics anxiety with other anxiety and attitude measures. For instance, is physics anxiety unique, and how is it related to different anxiety measures (e.g., math anxiety, science anxiety, test anxiety)?

\section{ACKNOWLEDGMENTS}

This material is based upon work supported by the National Science Foundation under Grant No. 1348614.
[1] A. Madsen, S. B. McKagan, and E. C. Sayre, Phys. Rev. ST Phys. Educ. Res. 9, 2 (2013).

[2] L. E. Kost, S. J. Pollock, and N. D. Finkelstein, Phys. Rev. ST Phys. Educ. Res. 5, 1 (2009).

[3] M. Lorenzo, C. H. Crouch, and E. Mazur, Am. J. Phys. 74, 118 (2006).

[4] P. B. Kohl and H. V. Kuo, AIP Conf. Proc. 1179, 173 (2009).

[5] A. Miyake et al., Science 330, 6008 (2010).

[6] R. Hembree, J. Res. Math. Educ. 21, 1 (1990).

[7] J. V. Mallow, Am. J. Phys. 46, 862 (1978).

[8] I. M.Lyons and S. L. Beilock, Cereb. Cortex 22, 9 (2012).

[9] I. M. Lyons and S. L. Beilock, PLoS ONE 7, 10 (2012).
[10] A. Devine et al., Behav. Brain Funct. 8, 33 (2012).

[11] J. V. Mallow, J. Sci. Educ. Technol. 3, 4 (1994).

[12] M. K. Udo, G. P. Ramsey, and J. V. Mallow, J. Sci. Educ. Technol. 13, 4 (2004).

[13] M. K. Udo et al., J. Sci. Educ. Technol. 10, 3 (2001).

[14] M. Sahin, S. Caliskan, and U. Dilek, Int. J. Env. Sci. Ed. 10, 2, (2015).

[15] M. Sahin, J. Balt. Sci. Educ. 13, 2 (2014).

[16] A. Gungor, A. Eryilmaz, and T. Fakioglu, J. Res. Sci. Teach. 44, 8 (2007).

[17] B. R. Wilcox and H. J. Lewandowski, Phys. Rev. Phys. Educ. Res. 12, 2 (2016). 